\title{
La teoría de los campos conceptuales y su papel en la enseñanza de las matemáticas
}

\section{The conceptual fields theory and its role in Mathematics Education}

\author{
Cristian Alfaro-Carvajal \\ cristian.alfaro.carvajal@una.cr \\ Escuela de Matemática \\ Universidad Nacional \\ Heredia, Costa Rica \\ Jennifer Fonseca-Castro \\ jennifer.fonseca.castro@una.cr \\ Escuela de Matemática \\ Universidad Nacional \\ Heredia, Costa Rica
}

Recibido-Received: 2/mar/2015 / Aceptado-Accepted: 6/may/2015 / Publicado-Published: 31/ene/2016.

\begin{abstract}
Resumen
Este artículo hace referencia a la teoría de campos conceptuales de Vergnaud y sus implicaciones en la enseñanza de las matemáticas. Se examinan conceptos fundamentales de dicha teoría a la luz de la enseñanza y aprendizaje de las matemáticas; dando ejemplos concretos en la disciplina y estableciendo relación con otros referentes relacionados, por ejemplo, resolución de problemas de Polya, situaciones didácticas de Brousseau, entre otros.

Palabras claves: Campos conceptuales, enseñanza de las matemáticas, esquemas.

Abstract

This article refers to the theory of Vergnaud's conceptual fields and its implications in teaching mathematics. Fundamental concepts of this theory are discussed in light of teaching and learning mathematics; providing specific examples in the discipline and establishing relationship with other related references; for example, Polya problem-solving and Brousseau didactic situations, among others.
\end{abstract}

Keywords: conceptual fields; mathematics education; scheme

La concepción de la educación matemática y la orientación que se dé a los procesos de enseñanza y aprendizaje dependen de una gama muy compleja de factores; entre estos, la concepción que se adopte sobre las matemáticas y la interpretación que se acepte de la adquisición de las operaciones y estructuras lógico-matemáticas (Piaget et. al., 1978).

En efecto, se pueden considerar las matemáticas a priori y adoptar un punto de vista platónico, en el cual los entes matemáticos existen independientemente del sujeto y son reducidos a un lenguaje sin ninguna referencia del mundo. Desde esta perspectiva, se justificaría plenamente el ocuparse principalmente de la simple transmisión de las verdades a quien se enseña por parte del profesorado, utilizando el lenguaje formal sin preocuparse de las ideas de sus estudiantes. 
Por otra parte, hay quienes asumen que las matemáticas son conocimientos de las percepciones humanas sobre el mundo y, como todo conocimiento, surge en una relación entre el sujeto y el objeto. Estas son entendidas como una combinación de elementos del "mundo exterior al sujeto pero, también de sus acciones y operaciones" (Ruiz, 2000, p. 56).

De acuerdo con esta postura, la enseñanza de las matemáticas debe verse como un proceso de construcción a través del planteamiento de situaciones didácticas, en las cuales el alumnado debe efectuar un trabajo similar al de la persona matemática, con un papel activo y donde la resolución de problemas debe estar siempre presente como propuesta didáctica (Brousseau, 1986).

Desde esta perspectiva y para el análisis de las situaciones didácticas, es fundamental la teoría de campos conceptuales de Gérard Vergnaud (1990), la cual proporciona un marco de referencia para el desarrollo y aprendizaje de competencias complejas. Para Vergnaud (1982), el conocimiento está organizado en "campos conceptuales cuyo dominio, por parte del sujeto, ocurre a lo largo de un extenso período de tiempo, a través de la experiencia, madurez y aprendizaje" (p. 40).

El objetivo de este artículo es discutir las principales ideas de la teoría de los campos conceptuales y sus implicaciones en la enseñanza y aprendizaje de las matemáticas. Se hace relación de dicha teoría con otros referentes importantes tales como la teoría de situaciones didácticas de Guy Brousseau y la resolución de problemas de Polya.

En las secciones siguientes se discuten los conceptos claves de esta teoría tales como concepto, esquema, situación, así como el concepto mismo de campo conceptual, retomando aspectos generales de estos y examinándolos a la luz de la enseñanza de las matemáticas.

\section{Campos conceptuales}

Un campo conceptual es un conjunto informal y heterogéneo de problemas, situaciones, conceptos, relaciones, estructuras, contenidos y operaciones del pensamiento, conectados unos a otros y, probablemente, entrelazados durante el proceso de aprendizaje. No es una teoría de enseñanza de conceptos explícitos y formalizados. Estudia procesos de conceptualización de lo real que permitan visualizar continuidades y rupturas entre conocimientos desde el punto de vista de su contenido conceptual (Vergnaud, 1990).

En consecuencia, es una teoría compleja, pues trata de involucrar en una misma teoría todo el desarrollo de situaciones progresivamente controladas, de conceptos y teoremas necesarios para operar eficientemente en una situación, y de las palabras y símbolos que pueden representar eficazmente esos conceptos y operaciones para el estudiantado, dependiendo de sus niveles cognitivos (Vergnaud, 1994).

Así, se puede entender un campo conceptual como un conjunto de situaciones entendidas como tareas. Esta definición genera una clasificación de los campos conceptuales que recae sobre el análisis de las tareas cognitivas y en los procedimientos que se ponen en juego en cada una de ellas. Por ejemplo, cuando el conjunto de situaciones planteadas requieren de una adición, una sustracción o una combinación de ambas, se está en el campo conceptual de las estructuras aditivas.

En principio, toda situación se puede analizar como una combinación de tareas de las que es importante conocer la naturaleza y la dificultad. En la teoría de los campos conceptuales, la complejidad de las tareas recae en los conceptos matemáticos y, por ende, la forma de los enunciados y el número de elementos puestos en juego tienen un rol secundario. En efecto, la 
ISSN Electrónico: 2215-3470

DOI: http://dx.doi.org/10.15359/ru.30-1.2
UNICIENCIA Vol. 30, No. 1, pp. 17-30. Enero-Junio, 2016.

URL: www.revistas.una.ac.cr/uniciencia Email: revistauniciencia@una.cr

primera entrada de un campo conceptual es la de las situaciones; y una segunda entrada es la de conceptos y teoremas (Vergnaud, 1990).

De acuerdo con el análisis de las tareas (situaciones), Vergnaud (1990) identifica los siguientes campos conceptuales:

- Estructuras aditivas. Incluyen situaciones que implican una o varias adiciones o sustracciones, así como los conceptos y teoremas asociados. Los conceptos relacionados son los de cardinal, medida, transformación temporal por aumento o disminución, relación de comparación cuantificada, composición binaria de medidas, etc. Algunos teoremas son:

$\operatorname{Card}(A \cup B)=\operatorname{Card} A+\operatorname{Card} B$ siempre que $A \cap B=\varnothing$

$A C=A B+B C$

- Estructuras multiplicativas. Son situaciones que implican una o varias multiplicaciones o divisiones, así como los conceptos y teoremas asociados. Los conceptos relacionados son proporción simple y múltiple, función lineal y n-lineal, razón escalar directa e inversa, cociente y producto de dimensiones, combinación lineal y aplicación lineal, fracción, razón, número racional, múltiplo y divisor, etc. Los teoremas incluyen las propiedades de isomorfismo de la función lineal y las propiedades referidas al coeficiente constante de dos variables linealmente ligadas.

$f(n x)=n f(x)$

$f\left(n_{1} x+n_{2} y\right)=n_{1} f(x)+n_{2} f(y)$

$f(x)=a x, x=\frac{1}{a} f(x)$

- La electricidad. Involucra situaciones como la iluminación de una habitación, la conexión de una lámpara a una pila, la comprensión del circuito eléctrico de una habitación. Conceptos de intensidad, tensión, resistencia y energía para los cálculos de electrocinética, etc.

- La mecánica. Incluye las magnitudes espaciales (longitudes, superficies, volúmenes). La conceptualización requiere de la geometría, las estructuras aditivas y las multiplicativas.

- La lógica de clases. Son situaciones que tienden a recubrir cuestiones para el desarrollo y el aprendizaje de la racionalidad. Conceptos de propiedad y característica, la relación de inclusión, operaciones de: intersección, unión, complementario sobre las clases y operaciones de conjunción, disyunción y de negación de estas propiedades. Teoremas tales como:

$A \subseteq B \Rightarrow \operatorname{Card} A \leq \operatorname{Card} B$

$\operatorname{Card}(A \cup B)=\operatorname{Card} A+\operatorname{Card} B-\operatorname{Card}(A \cap B)$

En la teoría de campos conceptuales tres asunciones son de gran relevancia: (1) un concepto no se forma dentro de un solo tipo de situaciones; (2) una situación no se analiza con un solo concepto; y (3) la construcción y apropiación de todas las propiedades de un concepto o de todos los aspectos de una situación es un proceso extenso que se extiende a lo largo de los años, 
con analogías y mal entendidos entre situaciones, conceptos, procedimientos y significantes (Vergnaud, 1983).

De lo anterior se destacan conceptos claves que permiten definir y entender dicha teoría, tales como, concepto, situación, significantes, entre otros. A continuación se explican y ejemplifican con detalle los principales conceptos de la teoría de campos conceptuales.

\section{Conceptos claves de la teoría de campos conceptuales}

Supóngase que se está interesado, como enseñantes, en que el estudiantado aprenda un concepto matemático determinado, para tal efecto, el concepto no debe ser reducido a una definición, sino más bien, deben plantearse situaciones-problemas con la idea de que el concepto tenga sentido para el alumnado. Esta afirmación existe en función del desarrollo de la inteligencia como una construcción espontánea y gradual de las estructuras lógico-matemáticas. Por lo tanto, en principio, no se puede limitar al plano del lenguaje y dejar de lado el papel de las acciones. Lo anterior es planteado desde la teoría de situaciones didácticas de Brousseau (1986), en las cuales, para el aprendizaje de un concepto, lo fundamental son los juegos-acciones del alumnado y, posteriormente, el lenguaje y las definiciones en la institucionalización por parte del profesorado.

En esta línea, se distinguen dos tipos de situaciones: (1) aquellas en las cuales el sujeto dispone, en un momento dado de su desarrollo y de las competencias para tratar fácilmente la situación propuesta; y (2) aquellas en donde no dispone de todas las competencias, lo cual le obliga a un proceso de reflexión, dudas, exploración, entre otros, que le conducirá al éxito o al fracaso.

En ambos casos, Vergnaud (1990) propone el papel de los esquemas. Para el primer caso, el esquema es único y se manifiesta en conductas muy automatizadas. En el segundo caso, se evocan varios esquemas que pueden competir entre sí y que, por lo tanto, deben ser acomodados, separados y recombinados.

Se le llama esquema "a la organización invariante de la conducta para una clase de situaciones dada” (Vergnaud, 1990, p. 2). De acuerdo con esto, pareciera que todos tienen un bagaje lógicomatemático que se pone en acción según la situación-problema que se enfrente. La noción de invariante supone cierta automatización de la conducta del individuo.

Desde la perspectiva de esta teoría, desde la niñez existen estructuras de pensamiento que se pueden llamar "nociones matemáticas". Es importante entender que estas estructuras son a nivel de pensamiento y no de lenguaje simbólico. Para Verganaud, estas estructuras son llamadas conocimientos en acto.

Estos conocimientos en acto están presentes en vivencias cotidianas. Se distinguen claramente dos tipos de experiencias ligadas a las acciones materiales del sujeto. Las primeras, las experiencias físicas que consisten en actuar sobre los objetos con el fin de descubrir propiedades que estos ya poseían antes de su manipulación por el sujeto; por ejemplo, el peso, la densidad, entre otros. Segundo, las experiencias lógico-matemáticas debido a la información que se obtiene a partir de los objetos particulares (físicos) y de las propias acciones que el sujeto ejerce sobre ellos.

Un ejemplo de esto es un niño pequeño de 4 o 5 años de edad, quien toma piedras en un jardín y las pone en fila para contarlas. Lo hace primero de izquierda a derecha y obtiene 10 en total. Después las cuenta de derecha a izquierda y obtiene con sorpresa el mismo número 10. Las pone ahora en círculo y obtiene otra vez 10 , tanto girando en un sentido como en otro. 
Obviamente, ni la suma ni el orden son algo propio de las piedras antes de que el sujeto las ponga en fila o las reúna todas; de esta forma el niño ha descubierto que la acción de reunir da resultados independientes de la de ordenar. Tenemos de este modo que las competencias matemáticas son sostenidas por esquemas organizadores de la conducta.

La pregunta ahora es, si estos conocimientos en actos y competencias matemáticas están presentes en todos los individuos desde la niñez, ¿cuál es el beneficio de este conocimiento en acto para la enseñanza de las matemáticas?, ¿cómo se pueden explotar estos conocimientos en acto?

La respuesta a la primer pregunta radica, precisamente, en que los conocimientos en acto que se podrían considerar anteriores a los conocimientos matemáticos elaborados en la institucionalización matemática, son fundamentales para lograr la conceptualización matemática y lograr un pensamiento deductivo, debido a que las coordinaciones de las acciones y las experiencias lógico-matemáticas dan lugar, al interiorizarse, al desarrollo de la abstracción necesaria en las matemáticas.

Esto lo plantea Vergnaud (1990) cuando afirma que en los esquemas están siempre presentes conocimientos llamados conceptos en acto y teoremas en acto o, en su forma más global, los invariantes operatorios. Así, por ejemplo, en un esquema de recuento se pueden identificar dos ideas matemáticas vitales para el funcionamiento del esquema: el de la biyección y el de cardinal. Por lo tanto, en la enseñanza debemos ser conscientes de la existencia de tales invariantes, ya que esto permite la reconstrucción del conocimiento por parte del alumnado.

La respuesta a la segunda pregunta, la ofrece Brousseau (1986) al afirmar que el trabajo del alumno debe ser, en ciertos momentos, comparable a la actividad científica de la persona matemática.

Saber matemáticas, no es solamente aprender definiciones y teoremas, para reconocer el momento de utilizarlos y aplicarlos; sabemos que aprender matemáticas implica ocuparse de problemas. Una buena reproducción por el alumno de una actividad científica exigiría que intervenga, que formule, que pruebe, que construya modelos, lenguajes, conceptos, teorías, que los intercambie con otros, que reconozca los que están conformes con la cultura, que tome los que son útiles, etc. (p. 6)

Es claro que los invariantes operatorios presentes en los esquemas de Vergnaud juegan un papel relevante en el rol del estudiantado que propone Brousseau, ya que se le considera a este último en un rol activo en el cual no espera que su profesor o profesora le transmita el conocimiento. Claramente, estos conceptos y teoremas en acto deben ser explotados a través de las situaciones didácticas. Es evidente, en este punto, la complejidad de la tarea de enseñante. Según Brousseau (1986), el personal docente debe crear situaciones para sus estudiantes, en las cuales los conocimientos aparecerán como la solución óptima a los problemas propuestos y sea el alumnado quien lo descubra.

Es importante señalar, como se hizo al inicio, que la visión de las matemáticas que tenga el personal docente es fundamental para su práctica en el aula. Solamente se puede adoptar una pedagogía en la que nuestras creencias estén realmente representadas. Por lo tanto, el planteamiento de situaciones que sus estudiantes puedan vivir, tiene que ver con las concepciones matemáticas que tenga quien ejerce la docencia. Si se acepta que las acciones del alumnado mueven esquemas que contienen conceptos en actos, será más simple entender que un concepto no se aprende solo por su definición. 
UNICIENCIA Vol. 30, No. 1, pp. 17-30. Enero-Junio, 2016.

ISSN Electrónico: 2215-3470

URL: www.revistas.una.ac.cr/uniciencia

Email: revistauniciencia@una.cr

El funcionamiento cognitivo del alumnado está conformado por operaciones que se automatizan progresivamente y de decisiones conscientes que permiten tener control de las condiciones en las cuales una operación es apropiada o no (Verganud, 1990). Por ejemplo, después de que el estudiantado aprende que,

$$
\begin{aligned}
& \frac{a+b}{c}=\frac{a}{c}+\frac{b}{c}, \quad(a b)^{n}=a^{n} b^{n}, \quad \sqrt{a b}=\sqrt{a} \sqrt{b} \text { extrapola a } \\
& \sqrt{a+b}=\sqrt{a}+\sqrt{b}, \quad \cos (a+b)=\cos a+\cos b .
\end{aligned}
$$

En casos como estos, es recomendable no corregir al estudiantado directamente, sino más bien, hacerlo entrar en contradicción mediante contra ejemplos. El poder que tiene el profesorado no tendrá un carácter sensor y prohibitivo como es usual en otras esferas, más bien debe ser un elemento que permita a sus estudiantes comprender el error (Chevallard, 1991).

Aunque se dé una automatización, esto no impide que se tomen decisiones conscientes. Por ejemplo, cuando se enseña el algoritmo de la adición no es posible explicitar todas las reglas necesarias para efectuar la operación; es decir, quedan elementos implícitos en los esquemas o mejor dicho una conceptualización implícita.

Hay que recordar que existen situaciones que el alumnado resuelve sencillamente usando un único esquema. No obstante, al plantearse una situación para la cual el alumnado no tiene todas las competencias matemáticas, acude a varios esquemas simultáneamente. Es decir, el funcionamiento cognitivo de un sujeto o de un grupo de sujetos en situación está basado en el repertorio inicial de los esquemas disponibles. Ideas que van de la mano con lo expuesto por Polya (1965) en su libro Cómo plantear y resolver problemas. En este, el autor afirma que para resolver un problema es necesario hacer uso de conocimientos y experiencias previas, así como de recordar teoremas y definiciones conocidas. A este proceso Polya lo llama "movilización". Sin embargo, para resolver un problema no basta recordar hechos aislados, hay que combinarlos entre sí adaptándolos al problema propuesto. Dicha actividad de adaptación y combinación la llama “organización”.

Acorde con lo anterior, aún con sus diferencias, la movilización y la organización son procesos que están ligados a la combinación de los esquemas de Vergnaud. Para este último, hay numerosos ejemplos de esquemas en el aprendizaje de las matemáticas y cada uno de ellos es relativo a una clase de situaciones cuyas características son bien definidas.

Un individuo puede aplicar un esquema a una clase más pequeña que la que se podría aplicar eficazmente, se tiene así un problema de extensión del esquema a una clase más amplia; y se puede hablar de deslocalización, generalización, transferencia, descontextualización. En este proceso se reconocen analogías entre la clase de situaciones sobre la cual el esquema era ya operatorio y las nuevas situaciones. La clave de la generalización del esquema es el reconocimiento de invariantes.

Para Polya (1965),

La generalización consiste en pasar del examen de un objeto, al examen de un conjunto de objetos, entre los cuales figura el primero; o pasar del examen de un conjunto limitado de objetos al de un conjunto más extenso que incluya al conjunto limitado. (p. 97) 
ISSN Electrónico: 2215-3470

DOI: http://dx.doi.org/10.15359/ru.30-1.2
UNICIENCIA Vol. 30, No. 1, pp. 17-30. Enero-Junio, 2016.

URL: www.revistas.una.ac.cr/uniciencia Email: revistauniciencia@una.cr

Polya ilustra la definición de generalización con el siguiente ejemplo:

Si se observa la suma $1+8+27+64=100$, la misma se puede expresar como

$$
1^{3}+2^{3}+3^{3}+4^{3}=10^{2} \text {. }
$$

Es natural preguntarse si la suma de cubos sucesivos es también un cuadrado; al hacer una pregunta así se está generalizando. Para responder a esto, se deben observar los invariantes operatorios.

También puede suceder que el individuo aplique un esquema a una clase demasiado amplia, de modo que el esquema falla, por lo tanto, debe restringir el alcance. Por ejemplo, al efectuar la suma $\frac{a}{b}+\frac{c}{d}$, es posible que una estudiante aplique el esquema de la multiplicación de fracciones, obteniendo como resultado $\frac{a}{b}+\frac{c}{d}=\frac{a+c}{b+d}$. En este caso, su docente debe ponerla en contradicción y plantearle la situación siguiente

$$
3+7=\frac{3}{1}+\frac{7}{1}=\frac{3+7}{1+1}=\frac{10}{2}=5 \text {. }
$$

De este modo se ve en práctica la productividad del poder que no sanciona, sino más bien, busca extraer la respuesta correcta.

El esquema es un concepto fundamental en psicología cognitiva y en didáctica, se reconoce que está compuesto de reglas de acción y de anticipaciones, pues genera una serie de acciones con el fin de lograr un cierto objetivo. Sin embargo, no siempre se reconoce que está compuesto de forma esencial de invariantes operatorios y de inferencias (Vergnaud, 1990). Estos últimos componentes son fundamentales para poner a funcionar el esquema en cada situación particular. Los invariantes operatorios son esencialmente de tres tipos lógicos: (1) los invariantes del tipo proposicional los cuales son susceptibles de ser falsos o verdaderos; (2) los de tipo función proposicional, que no son susceptibles de ser falsos o verdaderos, pero son indispensables para la construcción de proposiciones; y (3) de tipo argumento. En matemáticas, los argumentos pueden ser objetos materiales, personajes, números y proposiciones.

Esta clasificación de los invariantes operatorios tiene varias razones. La primera es clarificar que dichos invariantes no son de un tipo lógico único; y es necesario, por ende, analizar la condición particular de cada uno de ellos. La segunda razón es que un concepto en acto no es un concepto; ni un teorema en acto es un teorema. Estos últimos son apenas una pequeña parte visible de la conceptualización que, no obstante, sin los invariantes no serían nada. A su vez, no se puede hablar de invariantes operatorios ligados a los esquemas, sin la ayuda de las categorías del conocimiento explícitas: proposiciones, funciones proposicionales, objetos-argumentos.

Queda manifiesto que existe una relación dialéctica entre un concepto y los invariantes operatorios involucrados. Esto es central, ya que un concepto no podrá aprehenderse ajeno a las concepciones internas del individuo; la operacionalidad del concepto debe ser examinada por medio de situaciones diversas.

Esto impacta la forma en que se concibe la enseñanza de las matemáticas. De acuerdo con Vergnaud (1990), no se puede pretender presentar una definición al alumnado y que este la asimile como un concepto. 
Por ejemplo, Murillo, Soto, y Araya (2003, p. 102) afirman que una función se define de la siguiente manera:

Sea $D$ el conjunto de valores que puede tomar una variable independiente, y sea $C$ el conjunto de valores para la variable dependiente. Si $f$ es una regla de asociación entre los elementos de $D$ y $C$, entonces $D, C$ y $f$ determinan una función, si $f$ asigna a cada elemento $x \in D$ un único elemento $y \in C$ llamado $f(x)$ o imagen de $x$ bajo $f$.

Con esta definición, muchas veces el profesorado se frustra porque sus estudiantes no comprenden qué es el objeto sobre el cual trata dicha definición, pues se acude solo al aspecto simbólico, el cual como se tratará más adelante, es importante en la conceptualización matemática, pero claramente, no es suficiente.

Así, la investigación debe analizar una gran variedad de conductas y de esquemas para comprender en qué consiste, desde el punto de vista cognitivo, un concepto. Regresando al ejemplo anterior, el concepto de función no se comprende sino a través de una diversidad de problemas prácticos y teóricos.

Repetidas veces se ha utilizado el término concepto, pero no se ha clarificado su significado en la teoría de campos conceptuales, y este es de gran relevancia para entender dicha teoría. Vergnaud (1990) define un concepto como una tripleta que considera tres conjuntos, $C(S, I, \Gamma)$, en donde $S$ es el conjunto de situaciones que dan sentido al concepto, $I$ es el conjunto de invariantes sobre los cuales reposa la operacionalidad de los esquemas (el significado), y $\Gamma$ es el conjunto de las formas linguísticas y no linguísticas que permiten representar simbólicamente el concepto, sus propiedades, las situaciones y los procedimientos de tratamiento.

Lo anterior, vincula al personal docente de matemáticas a un reto enorme; al planteamiento de situaciones que proporcionen sentido al concepto en cuestión para sus estudiantes, sin reducir el significado de un concepto ni a los significantes ni a las situaciones.

Por su parte, una situación es aquella en donde los "procesos cognitivos y las respuestas del sujeto están en función de las situaciones a las que son confrontados" (Vergnaud, 1990, p. 10). En este sentido, se deben considerar dos aspectos. Primero, la variedad de situaciones a las que ha sido expuesto el sujeto en un campo conceptual. Segundo, la historia de situaciones que ha confrontado el sujeto y que le ha permitido dar sentido a los conceptos y procedimientos aprendidos. La comprensión de estos dos aspectos son necesarios en la elaboración y escogencia de situaciones didácticas en la labor docente. La primera requiere la separación de una situación en elementos más simples, y la segunda favorece la escogencia de situaciones funcionales.

La teoría de situaciones de Brousseau recobra importancia en la formación de conceptos, ya que las situaciones son un componente esencial de este. Según Vergnaud (1990),

La organización de una situación didáctica en un proyecto colectivo de investigación para la clase supone la consideración a la vez de las funciones epistemológicas de un concepto, de la significación social de los dominios de experiencia a los cuales hace referencia, los juegos de papeles entre los actores de la situación didáctica, resortes del juego, del contrato y de la transposición. (p. 14)

A su vez se hace visible la importancia de los campos conceptuales en la teoría de situaciones. Si tenemos claro en qué campo conceptual se está trabajando, el alumnado presentará regularidades 
asombrosas en la manera que aborda una determinada situación; es decir, mueve esquemas similares. Se cree que quizás por esta razón el matemático Polya consideró que es posible construir heurísticas para la resolución de problemas y menciona que "la heurística moderna trata de comprender el método que conduce a la solución de los problemas, en particular las operaciones mentales típicamente útiles en este proceso" (Polya, 1965, p. 102).

Las situaciones le dan sentido a los conceptos matemáticos, entendiendo dicho sentido como los esquemas evocados en el sujeto individual por la situación o por un significante (Vergnaud, 1990). Este no está en las situaciones mismas ni en las palabras o en los símbolos matemáticos.

Una situación dada o un simbolismo particular no evoca en un individuo todos los esquemas disponibles. Esto quiere decir, que no se puede tener el sentido de la adición a través de una situación particular de adición.

Vergnaud (1990) se planteó la siguiente pregunta: ¿qué funciones cognitivas es necesario atribuir al lenguaje y a las representaciones simbólicas en la actividad matemática? Según el autor, en la teoría de los campos conceptuales esta función es triple: (1) ayuda a la designación y por tanto a la identificación de los invariantes (objetos, propiedades, relaciones, teoremas); (2) ayuda en el razonamiento y la inferencia; y (3) ayuda en la anticipación de los efectos y de los fines, a la planificación, y al control de la acción.

Además de las funciones de comunicación y representación del lenguaje, existe una función adicional, la cual consiste en ayudar al pensamiento (Vergnaud, 1990). Así, un individuo acompaña su acción de una actividad lingüística cuando tiene necesidad de planificar y de controlar una serie de acciones insuficientemente dominadas.

Esta actividad lingüística es importante ya que favorece el descubrimiento de las relaciones pertinentes, la organización temporal de la acción y su función. Esto es analizado por Vergnaud en función del individuo que está en situación; sin embargo, para el personal docente se puede analizar un poco diferente. En efecto, cuando se explica algún procedimiento, por ejemplo, resolver la ecuación de primer grado $-3 x+4=-2$, claramente el profesorado domina la situación; pero es importante que la actividad lingüística que él realiza contribuya en el alumnado al descubrimiento de las relaciones pertinentes.

Es necesario aclarar que la función del lenguaje en la representación se puede subdividir a su vez, en tres funciones las cuales son: la representación de los elementos pertinentes de la situación, la representación de la acción y la representación de las relaciones entre la acción y la situación (Vergnaud, 1990).

El lenguaje en su función representativa debe normar y ordenar el pensamiento estudiantil. La pregunta que surge es, ¿cuál es la función del profesorado en este proceso? Sin duda debe contribuir a que el alumnado logre captar las informaciones pertinentes, descomponer en etapas, analizar e inferir, entre otros.

Esto plantea una interrogante mayor, ¿cómo el profesorado contribuye con el alumnado? En efecto, este trabajo del alumnado parece interno a él; es decir, debe seleccionar los elementos pertinentes, conjeturar, descomponer la tarea en subtareas. El método de Polya (1965) usado con cuidado por el personal docente puede facilitar este proceso.

Por ejemplo, se plantea a un estudiante, sea de primaria, secundaria o de nivel superior, un problema o situación de demostración como el siguiente: 
UNICIENCIA Vol. 30, No. 1, pp. 17-30. Enero-Junio, 2016.

Email: revistauniciencia@una.cr

Dos ángulos están situados en dos planos diferentes, pero cada uno de los lados de uno es paralelo al lado correspondiente del otro, y en la misma dirección. Demostrar que los dos ángulos son congruentes. (Polya, 1965, p. 43)

De entrada, se hace patente la necesidad del lenguaje en su función representativa. El alumno debe seleccionar la información pertinente, entendida como argumentos, propiedades, teoremas y dirigir sus operaciones de pensamiento en términos de conjeturas y rechazo o aceptación. Se está de acuerdo en que la tarea es del alumno; pero ¿cómo el profesor puede dar herramientas útiles al estudiante? En este caso Polya $(1965$, p. 43). recrea una conversación ficticia entre un profesor y su alumno,

Profesor: ¿Cuál es la hipótesis?

Alumno: Dos ángulos están situados en diferentes planos. Cada uno de los lados de uno es paralelo al lado correspondiente del otro y en la misma dirección.

Profesor: ¿Cuál es la conclusión?

Alumno: Los ángulos son congruentes

Profesor: Dibuje una figura. Introduzca una notación apropiada (función del lenguaje).

En esta situación se espera que el estudiante realice una figura como la siguiente.

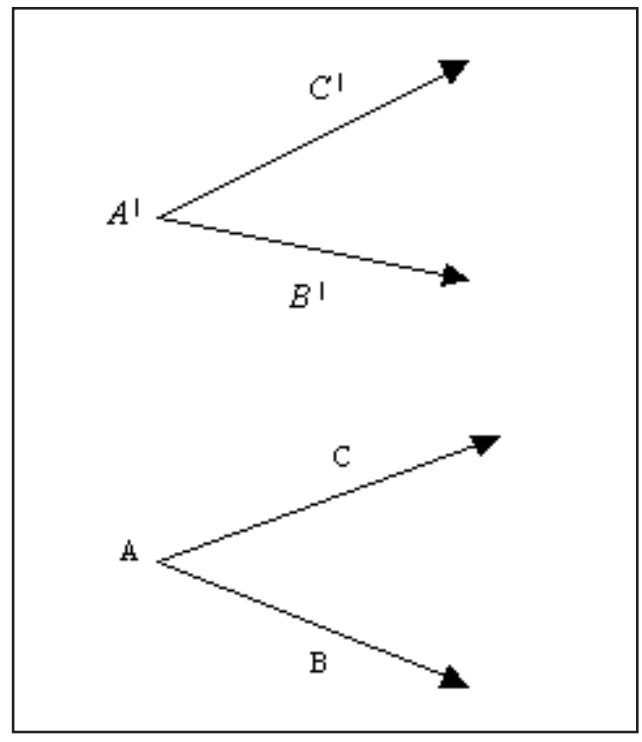

Figura 1. Primer bosquejo del problema de ángulos (Polya, 1965, p. 43). 
ISSN Electrónico: 2215-3470

DOI: http://dx.doi.org/10.15359/ru.30-1.2
UNICIENCIA Vol. 30, No. 1, pp. 17-30. Enero-Junio, 2016.

URL: www.revistas.una.ac.cr/uniciencia Email: revistauniciencia@una.cr

Profesor: ¿Cuál es la hipótesis? Le ruego que la formule empleando su notación (función de representación del lenguaje)

Alumno: $A, B, C$ no están en el mismo plano que $A^{\mid}, B^{\mid}, C^{\mid}$. Se tiene que $\overline{A B} / / \overline{A^{\mid} B^{\mid}}, \overline{A C} / / \overline{A^{\mid} C^{\mid}}$. Además, $\overline{A B}$ tiene la misma dirección que $\overline{A^{\mid} B^{\mid}}$y $\overline{A C}$ la misma dirección que $\overline{A^{\mid} C^{\mid}}$

Profesor: ¿Cuál es la conclusión?

Alumno: $\angle B A C \cong \angle B^{\mid} A^{\mid} C^{\mid}$

Profesor: Observe bien la conclusión y trate de pensar algún teorema que le sea familiar y que tenga la misma conclusión o una conclusión similar (sentido: esquemas evocados por el sujeto).

Alumno: Si dos triángulos son congruentes, sus ángulos correspondientes son congruentes

Profesor: Muy bien. He aquí, pues, un teorema relacionado con el propuesto y que ha sido demostrado ya. ¿Puede usted emplearlo?

Alumno: Creo que sí, pero no veo bien cómo.

Profesor: ¿Le haría falta introducir un elemento auxiliar para poder utilizarlo?... Veamos. El teorema que usted ha enunciado tan bien se refiere a triángulos, una pareja de triángulos congruentes. ¿En la figura dispone de triángulos?

Alumno: No, pero puedo hacer que figuren. Uniendo $B$ y $C, B^{\mid} y C^{\mid}$. Se tienen entonces dos triángulos $\triangle B A C \cong \triangle B^{\mid} A^{\mid} C^{\mid}$.

Profesor: Perfecto, pero, ¿de qué servirán esos triángulos?

Alumno: Para demostrar la conclusión, es decir, que $\angle B A C \cong \angle B^{\mid} A^{\mid} C^{\mid}$.

Profesor: Bien. Si es eso lo que quiere usted demostrar, ¿qué tipo de triángulos necesita?

Alumno: Triángulos iguales. Sí, claro está, yo puedo elegir $B, C, B^{\mid}, C^{\mid}$tal que $A B=A^{\mid} B^{\mid}$ y $A C=A^{\mid} C^{\mid}$. 
Email: revistauniciencia@una.cr

El estudiante dibuja algo parecido a la figura 2.

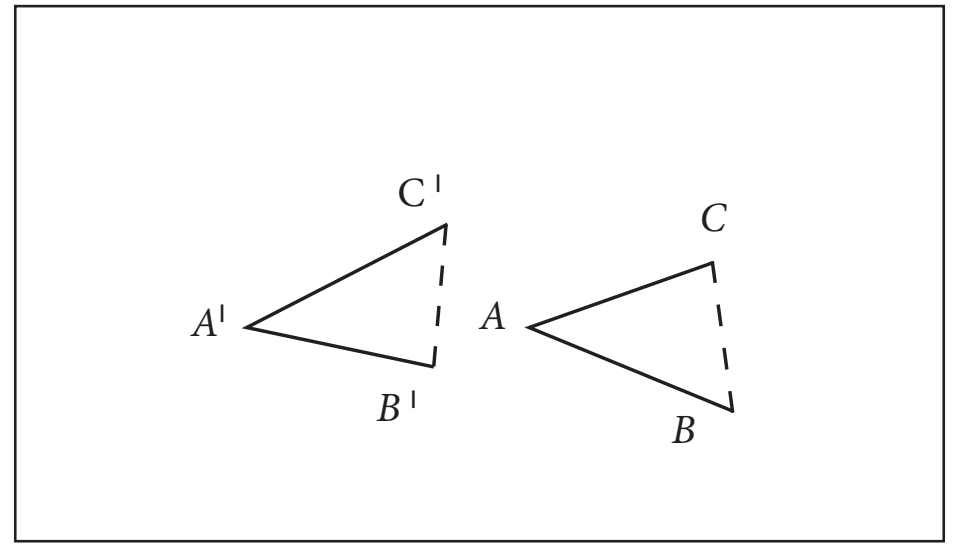

Figura 2. Segundo bosquejo del problema de ángulos (Polya, 1965, p. 44).

Profesor: Muy bien. Y ahora, ¿qué quiere demostrar?

Alumno: Quiero demostrar que los triángulos son iguales: $\triangle B A C \cong \triangle B^{\mid} A^{\mid} C^{\mid}$. Si lo puedo demostrar, la conclusión $\angle B A C \cong \angle B^{\mid} A^{\mid} C^{\mid}$sigue inmediatamente.

Profesor: Muy bien. Ahora se propone un nuevo fin, busca una nueva conclusión.

Pareciera plausible que esta forma de interrogación por parte del maestro hacia el alumno, contribuye favorablemente a que ordene sus operaciones de pensamiento.

Existe en el lenguaje natural medios de transformar verbos en sustantivos tales que los conceptos-herramienta se convierten en conceptos-objetos (Vergnaud, 1990). Por ejemplo, en la relación de orden en el conjunto de los números reales se puede usar varias expresiones para "menor que", por ejemplo, 3 pierde con 5, 5 le gana a 3, etc. Sin embargo, el simbolismo es útil ya que la invarianza del significante contribuye a la mejor identificación del significado y su transformación en objeto de pensamiento. Se puede esquematizar lo anterior con la figura 3.

\section{Lenguaje natural \\ Significante}

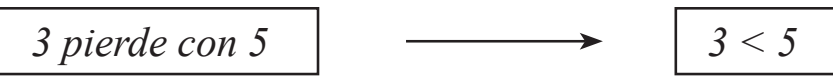

Figura 3. Lenguaje natural vs significante. Elaboración propia. 
ISSN Electrónico: 2215-3470

DOI: http://dx.doi.org/10.15359/ru.30-1.2
UNICIENCIA Vol. 30, No. 1, pp. 17-30. Enero-Junio, 2016.

URL: www.revistas.una.ac.cr/uniciencia Email: revistauniciencia@una.cr

Aunque el simbolismo contribuye a identificar un significado, queda claro, que él por sí mismo no determina un concepto; además, la pertinencia del simbolismo y del lenguaje es relativa a los conocimientos y al desarrollo cognitivo del alumnado.

\section{Consideraciones finales}

Las ideas expuestas a lo largo de este artículo muestran claramente que el aprendizaje de conceptos en matemáticas es complejo, ya que una reducción a definiciones no garantiza de modo alguno el aprendizaje. Esto supone la construcción de situaciones didácticas que permitan organizar un proceso interactivo y reflexivo por el profesorado implicado con sus estudiantes. Por lo tanto, la labor docente va más allá de transmitir conocimiento, lo cual concuerda con la teoría de situaciones didácticas de Brousseau.

No se puede teorizar sobre el aprendizaje de las matemáticas, ni partir solo del simbolismo o solo de las situaciones. Por lo tanto, es necesario considerar el sentido de las situaciones y de los símbolos. De este modo, se debe considerar al sujeto en situación y la organización de su conducta. Es aquí en donde se ven algunas similitudes con la propuesta de Polya en la resolución de problemas.

Se hace patente, también, la importancia concedida al desarrollo cognitivo del sujeto, en este punto se establece convergencia con las ideas de Piaget.

La concepción de campos conceptuales propuesta permite organizar las situaciones planteadas para los procesos de enseñanza y aprendizaje de las matemáticas de formas más coherentes, teniendo en cuenta los invariantes operatorios. Asimismo, es fundamental tener claro que un concepto no se desarrolla con un solo tipo de situaciones, una situación no se analiza con solo un concepto y la construcción y apropiación de un concepto es un proceso extenso y continuo a través del tiempo. Lo anterior implica una modificación en la manera de abordar la enseñanza y aprendizaje de las matemáticas.

Se coincide con Vergnaud en que el lenguaje, además de sus funciones de comunicación y representación, también es relevante en una función de ayuda al pensamiento. En efecto, es posible que el personal docente domine una situación, pero al exponerla debe acompañarla de una actividad lingüística que contribuya con el descubrimiento de relaciones por parte de sus estudiantes. No obstante, el alcance del término lenguaje utilizado por este autor es limitado, ya que la última función recae sobre el habla. En el interaccionismo simbólico, el lenguaje es considerado como un moldeador activo de la experiencia, el habla, por ejemplo, describe una práctica social, sirviendo en la comunicación para señalar experiencias compartidas y para la orientación en la misma cultura, más que un instrumento para el transporte directo del sentido.

\section{Referencias}

Brousseau, G. (1986). Fondements et méthodes de la didactiques des mathématiques. Recherches en Didactique des Mathématiques, 7(2), 33-115. Recuperado de http://cimate.uagro.mx/ivanlopez/seminario/archivos/Brousseau Fondements.pdf

Chevallard, Y. (1991). La transposición didáctica, Del saber sabio al saber enseñado. Buenos Aires: Aique Grupo Editor.

Murillo, M.; Soto, A.; y Araya, J. (2003). Matemática básica con aplicaciones. San José: Editorial Universidad Estatal a Distancia.

Piaget, J.; Inhelder, B.; Sinclair-de Zwart, H.; Cheret, M.; Revello, A. (1978). Memoria e intelligencia. Buenos Aires: El Alteneo. 
UNICIENCIA Vol. 30, No. 1, pp. 17-30. Enero-Junio, 2016.

ISSN Electrónico: 2215-3470

URL: www.revistas.una.ac.cr/uniciencia

DOI: http://dx.doi.org/10.15359/ru.30-1.2

Email: revistauniciencia@una.cr

Polya, G. (1965). Cómo plantear y resolver problemas. México: Editorial Trillas.

Ruiz, A. (2000). El desafío de las matemáticas. Heredia, Costa Rica: Editorial de la Universidad Nacional.

Vergnaud, G. (1982). A classification of cognitive tasks and operations of thought involved in addition and subtraction problems. En Carpenter, T., Moser, J. y Romberg, T. (edits.), Addition and subtraction: A cognitive perspective, pp. 39-59. Hillsdale, N. J.: Lawrence Erlbaum.

Vergnaud, G. (1983). Quelques problèmes theóriques de la didactique a propos d'un example: les structures additives. Atelier International d'Eté: Récherche en Didactique de la Physique. La Londe les Maures, Francia.

Vergnaud. G. (1990). La théorie des champs conceptuels. Récherches en Didactique des Mathématiques, 10(23), 133-170. Recuperado de http://www.fundesuperior.org/Articulos/Pedagogia/Teoria campos conceptuales.pdf

Vergnaud, G. (1994). Multiplicative conceptual field: what and why? En H. Guershon y J. Confrey (Eds.), The development of multiplicative reasoning in the learning of mathematics, pp. 41-59. Albany, N.Y.: State University of New York Press.

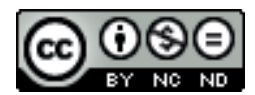

La Teoría de los Campos Conceptuales y su papel en la Enseñanza de las Matemáticas (Cristian Alfaro-Carvajal y Jennifer Fonseca-Castro) por Revista Uniciencia se encuentra bajo una Licencia CreativeCommons Atribución-NoComercial-SinDerivadas 3.0 Unported. 